Song et al., Afr J Tradit Complement Altern Med. (2014) 11(2):469-474

http://dx.doi.org/10.4314/ajtcam.v11i2.35

\title{
ELECTROACUPUNCTURING AT ZUSANLI POINT (ST36) ATTENUATES PRO-INFLAMMATORY CYTOKINE RELEASE AND ORGAN DYSFUNCTION BY ACTIVATING CHOLINERGIC ANTI-INFLAMMATORY PATHWAY IN RAT WITH ENDOTOXIN CHALLENGE
}

\author{
Qi Song ${ }^{1^{*}}$, Sen $\mathrm{Hu}^{2 \triangle}$, Haibin Wang ${ }^{2}, \mathrm{Yi} \mathrm{Lv}^{2}$, Xian $\mathrm{Shi}^{3}$, Ziyong Sheng ${ }^{2}$, Wei Sheng ${ }^{4 *}$
}
${ }^{1}$ The Oncotherapy center of the People's Liberation Army General Hospital, Beijing 100853 PRC. ${ }^{2}$ The Burns Institute, the First Hospital Affiliated to the General Hospital of People's Liberation Army, Beijing 100853 PRC. ${ }^{3}$ The
Department of acupuncture of the People's Liberation Army General Hospital, Beijing 100853 PRC. ${ }^{4}$ The
Cardiovascular surgery department of the People's Liberation Army General Hospital, Beijing 100853 PRC.
* These two authors contributed to the work equally and should be regarded as co-first authors.
${ }^{\triangle} \mathrm{Hu}$ Sen: corresponding author, the Burns Institute, the First Hospital Affiliated to the General Hospital of People's
Liberation Army, 51 Fu Cheng Road, Beijing, China 100048.

*E-mail: $\underline{\text { hs82080@163.com }}$

\begin{abstract}
Background: It is considered that the pathogenesis is closely related to an excessive production of pro-inflammatory cytokines caused by bacterial toxins and an imbalance between pro-inflammatory and anti-inflammatory mediators

Materials and Methods: This work investigates the effect of electro-acupuncturing (EA), at Zusanli point (ST36) on plasma cytokine release and organ dysfunction and their mechanism in conscious rats with endotoxin challenge.

Results: EA at Zusanli points obviously lowered the elevated levels of plasma TNF- $\alpha$, and attenuated changes in parameters relevant to various organ functions at $2 \mathrm{~h}$ after LPS challenge. a-BGT injection or bilateral cervical vagotomy could weaken or eliminate the effects of EA, and further aggravated the elevated levels of pro-inflammatory cytokines and organ dysfunction.

Conclusion: The results suggested that EA at Zusanli points significantly reduced the release of pro-inflammatory cytokines and organ dysfunction after LPS challenge by activating cholinergic anti-inflammatory pathway.
\end{abstract}

Key Words: Electro-acupuncture, Lipopolysaccharide, Cytokine, Cholinergic, anti-inflammatory pathway.

\section{Introduction}

Systemic inflammatory response and organ dysfunction induced by lipopolysaccharide is an important cause of sepsis, ending in MODS and death in injured patients with severe infections (Sheng and $\mathrm{Hu}, 1999$ ). At present, it is considered that the pathogenesis is closely related to an excessive production of pro-inflammatory cytokines caused by bacterial toxins and an imbalance between pro-inflammatory and anti-inflammatory mediators (Abraham et al., 2007). Therefore, to control the production of pro-inflammatory mediators, regulating the balance of neuron- endocrine-immune system could be considered an important strategy in preventing and controlling sepsis and MODS (Gentile and Singer, 2012). In 2000, the cholinergic anti-inflammatory pathway has been realized as an auto-regulation system of neuron-endocrine-immune, which can regulate or antagonize systemic inflammatory responses through the central nervous system via cholinergic nerve, and its neurotransmitter (Borovikova et al., 2000).

Previous studies showed that electrical stimulation of the vagus nerve and/or the administration of cholinomimetic drug carbachol could suppress levels of inflammatory cytokines in plasma, liver and small intestine; alleviate inflammatory response and pathological changes induced by endotoxin; ischemia of intestine, and animals with burn injury (Li et al., 2010; Setoguchi et al., 2011; Kox et al., 2012). However, it is difficult to apply electrical stimulation to vagus nerve in clinics due to complicated manipulation and untoward side effects, including serious tissue injury.

Acupuncture as one of therapeutic maneuvers in traditional Chinese medicine has been applied in clinics for thousands of years, and it has been found to have positive effect in regulating neuron-endocrine-immune system bi-directionally and antagonizing systemic inflammatory response with little side effects. Studies also showed that acupuncture of Zusanli (ST36), points, which was found to be related to parasympathetic system, could improve immunity and attenuate inflammatory response and hemorrhagic shock (Huo et al., 2003; Suo et al., 2010).

Since the effect of acupuncture at Zusanli (ST36) is similar to that of activating parasympathetic nerve, we hypothesize that the anti-inflammatory mechanism of acupuncture at bilateral Zusanli (ST36) is related to the activated cholinergic pathway, and its cholinergic receptor. Therefore, the objectives of our present study is to investigate the effects of EA at Zusanli (ST36) on plasma cytokine release and organ dysfunction in conscious rats with endotoxin challenge, and to explore the relationship between cholinergic anti-inflammatory pathway and the mechanism of EA which plays the role of anti-inflammatory effect and organ protection.

\section{Materials and methods Animal Model}

Seventy male Wistar rats (provided by Experimental Animal Center affiliated with Chinese Academy of Medical Sciences, weighing $(220 \pm 20) \mathrm{g}$ \}, were acclimated in our laboratory for one week, and they were fasted but allowed free access to water until 4 hours before the operation. To avoid the possible effect of anesthesia, the rats were allowed to claw into cloth bags by themselves without anesthesia before 
http://dx.doi.org/10.4314/ajtcam.v11i2.35

experiment. For all groups, rectal temperature was taken and WBC number was counted for self-control before and after endotoxin injection. LPS was given by intravenous injection ( $5 \mathrm{mg} / \mathrm{kg}$, Sigma, O111:B4).

\section{Acupoint and Intensity}

Both hind limbs were shaved and the skin was disinfected. ST36 acupuncture point, which was located at posterior and lateral side of the knee joint, $5 \mathrm{~mm}$ below capitulum fibulae ${ }^{10}$, was punctured with a depth of $7 \mathrm{~mm}$, and then the needle was connected with electro-acupuncture apparatus (HANS, made in china, LH202H). The electric current with the intensity of $2 \mathrm{~mA}, 2-100 \mathrm{~Hz}$ was continued for $1.5 \mathrm{~h}$. In groups of $\mathrm{VA}+\mathrm{EA}$ and $a$-BGT+EA, electro-acupuncture was applied $0.5 \mathrm{~h}$ after bilateral cervical vagotomy or $a$-bungarotoxin ( $a$-BGT, an antagonist of $a 7$ subunit of cholinergic $\mathrm{N}$ receptor) injection. Rats in LPS, VA control and a-BGT control groups were subjected to EA at sham acupoint points which were located at the bilateral capitulum fibulae apart from outside the fibulae, with the outside condyle center point to about $1 \mathrm{~cm}$ place.

\section{Groups and Treatments}

Rats were divided into 7 groups of 10 rats in each group according to the diagram of random number (Table 1).

\section{Measurement}

All animals were sacrificed $2 \mathrm{~h}$ after LPS injection. Arterial blood pressure, rectal temperature and peripheral WBC count were measured before sacrifice, and then blood and tissue samples were taken. Plasma TNF- $\alpha$ (Diaclone, France) and IL-10 (Biosource, USA) were measured with ELISA. ALT, and CK-MB were determined with automatic biochemical analyzer (HITACHI 7170, Japan). Serum diamine oxidase (DAO), activity was determined according to the method described by Xiao R, et al (Xiao et al., 2011). All reagents were purchased from Sigma (Aldrich, St. Louis, MO, USA).

\section{Statistical Analysis}

Data was presented as mean \pm SD. T test and F test were used to evaluate whether values at the same point were different between the control and treatment groups by SPSS10.0. A significant difference was presumed at a probability value less than 0.05 .

\section{Results}

Systemic manifestation: about 10 min after LPS injection, the rats showed piloerection, shivering, diarrhea, scrunching up, abdominal breathing (with acceleration of doubled respiratory rate), sensitiveness to external irritation. BP showed a temporary lowering after LPS injection followed by a slow rise 10 min later; both WBC count and rectal temperature lowered significantly after LPS injection.

The changes in cytokines (Figure 1 and 2): the concentrations of TNF- $\alpha$ and IL-10 in LPS group were significantly higher than those in sham group $(\mathrm{P}<0.01)$. EA of bilateral Zusanli points resulted in a significant decrease of concentration of TNF- $\alpha(\mathrm{P}<0.05)$, but there was no significant difference in level of IL-10 ( $>0.05)$. The levels of TNF- $\alpha$ and IL-10 in VA and $a$-BGT group were significantly higher than those in sham, LPS and LPS+EA groups $(\mathrm{P}<0.05)$. EA at ST36 didn't decrease the concentration of TNF- $\alpha$ and IL-10 after bilateral cervical vagotomy or $a-B G T$ injection.

Parameters of organ function (Table 2): ALT, CK-MB, Cr and DAO concentrations were significantly higher in all groups with LPS injection than those in sham group $(\mathrm{P}<0.05)$. EA at ST36 could significantly lower the levels of ALT, CK-MB, Cr and DAO in plasma $(\mathrm{P}<0.05)$. Bilateral cervical vagotomy and blockage of a7 subunit could aggravate organ dysfunction, whereas EA had no effect on ALT, CK-MB, Cr and DAO contents at that time.

Rates of tissue water content of heart, liver, kidney and intestine( Table 3): compared with sham control group, LPS injection resulted in high rates of tissue water content, denoted inflammation and edema $(\mathrm{P}<0.05)$. EA at ST36 could significantly lowered the rates of tissue water content of heart, liver, kidney and intestine to attenuate inflammation and edema $(\mathrm{P}<0.05)$, but had no effect when vagotomy and $a$-BGT injection.

Rates of tissue water content differed significantly $(\mathrm{P}<0.05)$ between sham group and LPS group, EA could significantly decrease rates of tissue water content $(\mathrm{P}<0.05)$, but had no effect when vagotomy or $a$-BGT injection. $* \mathrm{P}<0.05$ vs. sham group; \# $\mathrm{P}<0.05$ vs. LPS group

Conclusion The results suggested that EA at Zusanli points significantly reduced the release of pro-inflammatory cytokines and organ dysfunction after LPS challenge by activating cholinergic anti-inflammatory pathway. 
Song et al., Afr J Tradit Complement Altern Med. (2014) 11(2):469-474

http://dx.doi.org/10.4314/ajtcam.v11i2.35

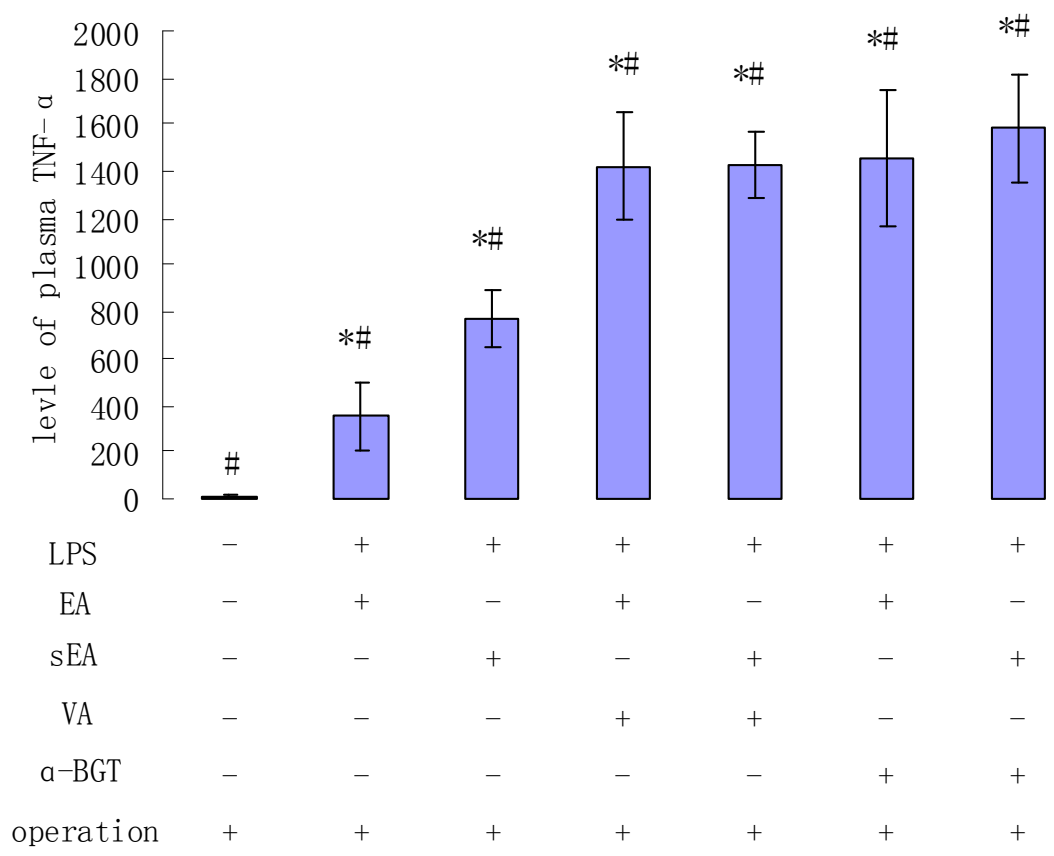

LPS injection resulted high level of TNF- $\alpha$, EA decreased TNF- $\alpha$ significantly than sEA, both vagotomy and $a$-BGT injection elevated TNF- $\alpha$ level further, EA had no effect of TNF- $\alpha$ when $a$-BGT injection or vagotomy. $\quad * \mathrm{P}<0.05$ vs. sham group; \# $\mathrm{P}<0.05$ vs. LPS group.

Figure 1: The change in plasma level of TNF- $\alpha$ in each group

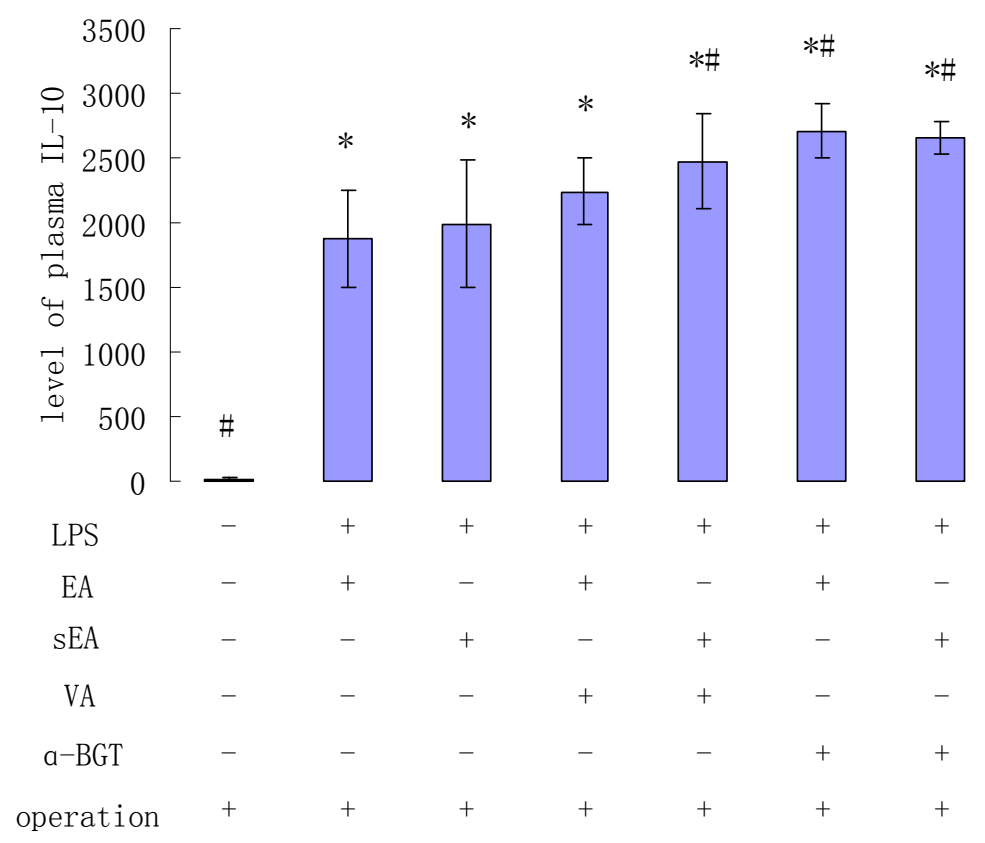

LPS injection resulted in high level of IL-10, both vagotomy and a-BGT injection elevated IL-10 level further, the level of IL-10 didn't differ significantly between EA and sEA treatment. $* \mathrm{P}<0.05$ vs. sham group; \# $\mathrm{P}<0.05$ vs. LPS group

Figure 2: The changes in plasma level of IL-10 in each group. 
Table 1: Rats were divided into 7 groups with 10 in each group according to the diagram of random number

\begin{tabular}{|c|c|c|c|c|c|c|}
\hline \multirow{2}{*}{ Groups } & \multicolumn{6}{|c|}{ Treatments } \\
\hline & LPS & EA & shamEA & Vagotomy & $\alpha$-BGT & Operation \\
\hline Sham & - & - & - & - & - & + \\
\hline EA & + & + & - & - & - & + \\
\hline LPS & + & - & + & - & - & + \\
\hline $\mathrm{VA}+\mathrm{EA}$ & + & + & - & + & - & + \\
\hline VA & + & - & + & + & - & + \\
\hline$\alpha-\mathrm{BGT}+\mathrm{EA}$ & + & + & - & - & + & + \\
\hline$\alpha$-BGT & + & - & + & - & + & + \\
\hline
\end{tabular}

All groups were subjected to different treatment.

sham, sham control; EA, electro-acupuncture; LPS, lipopolysaccharide; VA+EA, vagotomy+ electro-acupuncture; VA, vagotomy; $\alpha$-BGT+EA, a-bungarotoxin+electro-acupuncture; $\alpha$-BGT, a-bungarotoxin.

Table 2: Parameters of function of heart, liver, kidney and intestine $(\bar{x} \pm s)$

\begin{tabular}{|c|c|c|c|c|}
\hline \multirow{2}{*}{$\begin{array}{r}\text { Group } \\
(\text { each } \mathrm{n}=10)\end{array}$} & \multicolumn{4}{|c|}{ Blood variables } \\
\hline & CK-MB(U/L) & ALT(U/L) & $\mathrm{Cr}(\mathrm{U} / \mathrm{L})$ & $\mathrm{DAO}(\mathrm{KU} / \mathrm{L})$ \\
\hline Sham & $387 \pm 135$ & $25.5 \pm 2.7$ & $48.3 \pm 5.7$ & $1.10 \pm 0.40$ \\
\hline EA & $705 \pm 124 * \#$ & $48.7 \pm 4.6^{* \#}$ & $95.7 \pm 8.4 * \#$ & $1.76 \pm 0.38 * \#$ \\
\hline LPS & $1228 \pm 430 *$ & $56.8 \pm 3.6^{*}$ & $139.9 \pm 11.6^{*}$ & $2.52 \pm 0.63^{*}$ \\
\hline $\mathrm{VA}+\mathrm{EA}$ & $1565 \pm 358 * \#$ & $68.3 \pm 5.9 * \#$ & $198.3 \pm 15.9$ *\# & $2.96 \pm 0.60 *$ \\
\hline VA & $1606 \pm 348 * \#$ & $65.6 \pm 4.7 * \#$ & $206.9 \pm 24.7 * \#$ & $3.20 \pm 0.66 * \#$ \\
\hline $\mathrm{a}-\mathrm{BGT}+\mathrm{EA}$ & $1673 \pm 439 * \#$ & $61.2 \pm 9.4^{*}$ & $158.2 \pm 16.0$ *\# & $3.16 \pm 0.71$ *\# \\
\hline a-BGT & $2031 \pm 424 * \#$ & $56.1 \pm 16.2 *$ & $168.5 \pm 12.5$ *\# & $3.04 \pm 0.58^{*}$ \\
\hline
\end{tabular}

LPS injection resulted in high level of CK-MB, ALT, Cr and DAO. EA attenuated organ dysfunction significantly (all P<0.05), but had no organ protective effect when vagotomy or a-BGT injection. ${ }^{*} \mathrm{P}<0.05$ vs. sham group; \# $\mathrm{P}<0.05$ vs. LPS group.

Table 3: Rates of tissue water content of heart, liver, kidney and intestine $(\bar{x} \pm s$ )

\begin{tabular}{cllll}
\hline $\begin{array}{c}\text { Group } \\
\text { (each n=10) }\end{array}$ & Heart & Liver & kidney & Intestine \\
\hline Sham & $63.7 \pm 2.5$ & $62.5 \pm 2.0$ & $64.4 \pm 1.8$ & $65.6 \pm 2.6$ \\
EA & $69.1 \pm 2.4^{* \#}$ & $68.8 \pm 4.2^{* \#}$ & $67.6 \pm 3.7 \#$ & $67.4 \pm 2.6 \#$ \\
LPS & $74.7 \pm 2.8^{*}$ & $75.7 \pm 2.8^{*}$ & $75.8 \pm 4.2^{*}$ & $73.6 \pm 2.5^{*}$ \\
VA+EA & $80.7 \pm 3.6 \#$ & $83.7 \pm 3.8^{* \#}$ & $81.3 \pm 3.6^{* \#}$ & $78.8 \pm 2.8^{* \#}$ \\
VA & $81.9 \pm 4.4 \#$ & $84.9 \pm 4.6^{*} \#$ & $82.2 \pm 3.4^{* \#}$ & $79.9 \pm 3.0^{* \# .}$ \\
a-BGT+EA & $77.7 \pm 5.6^{*}$ & $73.7 \pm 3.9^{*}$ & $80.7 \pm 4.6^{*}$ & $83.2 \pm 5.1^{* \#}$ \\
a-BGT & $78.1 \pm 6.4^{*}$ & $74.9 \pm 5.6^{*}$ & $78.5 \pm 4.8^{*}$ & $82.7 \pm 4.8^{* \#}$ \\
\hline
\end{tabular}

LPS injection resulted in high level of CK-MB, ALT, Cr and DAO. EA attenuated organ dysfunction significantly (all P<0.05), but had no organ protective effect when vagotomy or $a-B G T$ injection. $* \mathrm{P}<0.05$ vs. sham group; \# $\mathrm{P}<0.05$ vs. LPS group

\section{Discussion}

The treatment of endo-toxemia with EA has rarely been reported, and one of the main reasons is lack of such an animal model for the study. Therefore, first of all we attempted to reproduce a model of EA in conscious rat with endotoxin challenge, and tried to prove its feasibility and effect with the following observations. (1) The changes in WBC count and body temperature after endotoxin injection according to the diagnostic criteria of animal SIRS as described by Sheng et al (Sheng and Hu, 1999). (2) Rats were allowed to enter black cloth bags by themselves, and then their four limbs were pulled out gently through the holes on the bag. The legs were then shaved to allow the performance of EA treatment in conscious state. (3) In the groups of bilateral vagotomy, the vagus nerve on each side was isolated under anesthesia (40mg/kg) first, then they were divided after rats recovered from anesthesia. (4) A modified intra-cutaneous imbedding needle, which could be fixed on the skin, was used 
http://dx.doi.org/10.4314/ajtcam.v11i2.35

for electric acupuncture of the ST36 point in the hind limb, which was considered as corresponding to Zusanli acupuncture point in humans. An electric current of $2 \mathrm{~mA}, 2-100 \mathrm{~Hz}$, was used to achieve an effective stimulation.

Nevertheless, our experiment showed that EA could lower the plasma level of TNF- $\alpha$ (pro-inflammatory cytokine) significantly in endoxemia rats, and had no significant effect on the plasma content of IL-10 (anti-inflammatory cytokine). The results suggested that EA at ST36 exerts its anti-inflammatory effect mainly by decreasing pro-inflammatory factor in plasma, but with little effect on anti-inflammatory factor. EA was also shown to be able to suppress the levels of CK-MB, ALT, Cr and DAO significantly, indicating that it was able to exert a protective effect on multiple organs in rats with endotoxemia. Recent studies showed that though LPS induced injury not only directly to multiple organs, but mainly by inflammatory mediators released from inflammatory cells induced by LPS resulting in injury to cardiac muscle cells and hepatic cells directly (Huo et al., 2003; Zhou et al., 2011). Therefore, blockade or decrease production of pro-inflammatory mediators by inflammatory cells should be considered as a main measure to reduce inflammatory response and protection of functional cells of the important organs from injury by LPS. Zusanli (ST36) is the confluent point of the Stomach Meridian of Foot-Yangming, and its principle therapeutic indication is for diseases of digestive system, by exerting the effects of dredging the excretory organs and immuno-regulation. It is considered in Traditional Chinese Medicine that dredging the excretory organs would result in a detoxification effect and preservation of Yin, by exerting an effect of detoxication and elimination of excessive unwholesome ferments. It was observed in our study that fetid diarrhea occurred in most of the rats receiving EA at ST36, and it might be the result of promotion of peristalsis of intestine by EA, and it was assumed that toxins were also expelled.

Borovikova (Borovikova et al., 2000), discovered that the systemic inflammation regulated pathway of cholinergic nerve and its neuro-transmitter-acetylcholine (ACh), be named as "cholinergic anti-inflammatory pathway," and it was considered that through this pathway, pro-inflammatory factors could be directly antagonized, and the lethal effect of endotoxin could be alleviated. It was further proved in further research that Ach exerted its anti-inflammatory effect via a7 subunit of cholinergic $\mathrm{N}$ receptor which participated in the post-transcription regulation (Wang et al., 2003). The application of electrical stimulation of vagus nerve has been limited in clinical treatment due to its complicated manipulation and untoward serious tissue injury, though it has been shown to have significant anti-inflammatory effect. On the other hand, acupuncture has been shown to have the effect of regulating neutron-endocrine-immune system bi-directionally and antagonize systemic inflammatory response. However, its mechanism is still not clear. Recent studies showed that EA at ST36 had a significant effect on dorsal nuclei of vagus nerve, solitary tract, nucleus of solitary tract and ambiguous nucleus, all of which were central nuclei of the vagus nerve (Liu et al., 2004). With the method of antrorse-retrorse electrical stimulation and intracellular recording, Zhuo Meng discovered that neurons of cornu dorsale medullae spinalis could receive afferent somatic messages from ST36, and they were transmitted to nucleus of solitary tract which is the splanchnic sensory nucleus, thus affecting the motor activity of the stomach and electrical release by efferent vagus nerve. EA at ST36 can also excite efferent vagus nerve fibers, accelerate the frequency of electrical discharge and increase the peak of its value (Ye et al., 2006).

Our study showed that EA at ST36 resulted in anti-inflammatory effect and organ protection, which maybe attributed to release of Ach by the vagus nerve as a result of transmission of message to vagus nuclei consequent on EA of ST36 acupoint. Therefore, bilateral division of vagus nerve significantly attenuates the anti-inflammatory and organ protection effect. Meantime, the balance of parasympathetic and sympathetic nerve to regulate systemic inflammation was lost, and the effect of sympathetic nerve impulse was strengthened and inflammatory response aggravated. Similarly, when a7 subunit of cholinergic N receptor was antagonized bya-BGT, EA of the ST36 point became ineffective. Therefore, it is our assumption that the cholinergic anti-inflammatory pathway is one of the main mechanisms of anti-inflammatory and organ protection as a result of EA at ST36. Though the mechanism of acupuncture of is very complicated, activation of cholinergic anti-inflammatory pathway may be considered to be one of the main mechanisms of EA at ST36 in exerting the effect of anti-inflammation and organ protection. Therefore, further studies are warranted to explore its relationship with other mechanisms, and whether there are also cross effects. Our study was limited in exploring the mechanism of traditional acupuncture with the aid of modern medical theory as to suggest a novel strategy for the treatment of sepsis, MODS and certain diseases due to excessive inflammatory reaction.

\section{Acknowledgement}

This work was supported by the special foundation of the 11 five-year plan for military medical project, No. $06 Z 055$

\section{References}

1. Abraham, E., and Singer, M. (2007). Mechanisms of sepsis-induced organ dysfunction. Crit Care Med. 35: 2408-2416.

2. Borovikova, L.V., Ivanova, S., Zhang, M., Yang, H., Botchkina, G.I., Watkins, L.R., Wang, H., Abumrad, N., Eaton, J.W., and Tracey, K.J. (2000). Vagus nerve stimulation attenuates the systemic inflammatory response to endotoxin. Nature. 405:458-462. 
Song et al., Afr J Tradit Complement Altern Med. (2014) 11(2):469-474

http://dx.doi.org/10.4314/ajtcam.v11i2.35

3. Gentile, L.F., Cuenca, A.G., Efron, P.A., Ang, D., Bihorac, A., McKinley, B.A., Moldawer, L.L., and Moore, F.A. (2012). Persistent inflammation and immunosuppression: A common syndrome and new horizon for surgical intensive care. J Tauma Acute Care Surg. 72:1491-1501.

4. Huo, Z.J., Zhang, L. and Qian, R.Q. (2003). Effects of Electro acupuncture of Different Acupoint Groups on Blood TNF- $\alpha$ IL-6、WBC and Cerebral Free Radicals in Cerebral Ischemia-Reperfusion Rats. Acupuncture Research. 28: 94-98.

5. Kox, M., Vaneker, M., van der Hoeven, J.G., Scheffer, G.J., Hoedemaekers, C.W., and Pickkers, P. (2012). Effects of Vagus Nerve Stimulation and Vagotomy on Systemic and Pulmonary Inflammation in a Two-Hit Model in Rats. PLoS One. 7: e34431.

6. Li, Z.R. (2003). Exp Acupuncture. China Press of Tradit Chinese Med., Beijing. Pp 327-328.

7. Li, Y.Z., Liu, X.H., Rong, F., Hu, S., and Sheng, Z.Y. (2010). Carbachol inhibits TNF- $\alpha$-induced endothelial barrier dysfunction through alpha nicotinic receptors Acta Pharmacol Sin. 31: 1389-1394.

8. Liu, J.H., Yan, J., Yi, S.X., Chang, X.R., Lin, Y.P., and Hu, J.M. (2004). Effects of electroacupuncture on gastric myoeletric activity and substance $P$ in the dorsal vagal comples of rats. Neurosci Lett.356: 99-102.

9. Setoguchi, D., Yatsuki, H., Sadahiro, T., Nakamura, M., Hirayama, Y., Watanabe, E., Tateishi, Y., and Oda, S. (2011). Effects of a peripheral cholinesterase inhibitor on cytokine production and autonomic nervous activity in a rat model of sepsis. Cytokine. 57: 238-244.

10. Sheng, Z.Y., and Hu, S. (1999). Multiple Organ Dysfunction Syndromes. Science Press, Beijing. Pp 11-61.

11. Suo, X.Y., Du, Z.H., Wang, H.S., Li, J.G., Wang, Y.L., Yao, S.D., and Chen, W.M. (2010).The effects of stimulation at acupoint ST36 points against hemorrhagic shock in dogs. Am J Emerg Med. 29: 1188-1193.

12. Wang, H., Yu, M., Ochani, M., Amella, C.A., Tanovic, M., Susarla, S., Li, J.H., Wang, H., Yang, H., Ulloa, L., Al-Abed, Y., Czura, C.J., and Tracey, K.J. (2003). Nicotinic acetylcholine receptora7 subunit is an essential regulator of inflammation. Nature. 421:384-388.

13. Xiao, R., Lei, Z.Y., Dang, Y. M., and Huang, Y.S. (2011). Prompt myocardial damage contributes to hepatic, renal, and intestinal Injuries soon after a severe burn in rats. J Trauma.71: 663-671.

14. Ye, X.F., Li, J.G., and Du, Z.H. (2006). Effect of Electroacupuncture at "Zusanli"(ST 36) on Vagal Electrical Activity in the Rat. Acupuncture Res. 31: 290-293.

15. Zhou, G., Hu, S., Lv, Y., Song, Q., Zou, X., and Sheng, Z. (2011). Carbachol allieviates rat cytokine release and organ dysfunction induced by lipopolysaccharide. J. Trauma. 71: 157-162. 